\title{
20.
}

\section{Ueber die Anwendung der Sturm'schen Methode auf transcendente Gleichungen.}

(Von Herrn Dr. Stern in Göttingen.)

Die Sturm'sche Methode der Auflösung der algebraischen Gleichungen wird gewöhnlich in folgender Gestalt vorgetragen. Ist eine algebraische Gleichung vom $m$ ten Grade

$$
\boldsymbol{F} \boldsymbol{x}=\mathbf{0}
$$

gegeben, so bildet man aus $F x$ und seinem Differentialquotienten $F_{1} x$ die Functionen $\stackrel{2}{\boldsymbol{F}} x, \stackrel{3}{\boldsymbol{F}} x$ u. s. w., bis man an die letzte $\stackrel{m}{\boldsymbol{F}} \boldsymbol{x}$ kommt. Diese Functionen sind durch die Gleichungen

$$
\begin{aligned}
& F x=A_{1} F_{1} x-F_{F}^{2} \\
& F_{1} x=A_{2} F^{2} x-F_{F}^{2} \\
& \cdot \cdot \cdot \cdot \cdot \cdot \cdot \cdot \cdot \cdot \\
& \stackrel{m_{-2}}{F} x=A_{m-1}{ }^{m-1} x-\stackrel{m}{F} x
\end{aligned}
$$

verbunden und es wird dabei vorausgesetzt, dass sowohl $F x$ und $F_{1} x$, als auch die folgenden Functionen nach den fallenden Potenzen von $x$ geordnet sind. Substituirt man in die Reihe $F x, F_{1} x \ldots . \mathscr{F}_{\boldsymbol{F}} \boldsymbol{x}$ statt $x$ den Werth $a$, und dann den grösseren Werth $b$, so wird die Zahl der reellen Wurzeln der Gleichung, die zwischen $a$ und $b$ liegen, der Zahl der Zeichenwechsel gleich sein, welche die dem Werthe $a$ entsprechende Zeichenreihe mehr enthält, als die dem Werthe $b$ entsprechende.

Es versteht sich von selbst, dass die Sturm'sche Methode in dieser Gestalt nicht auf transcendente Gleichungen anwendbar ist. Indessen hat schon Sturm selbst angedeutet, dass man auch den entgegengesetzten Weg einschlagen kann. Man schreibe nämlich $F x$ und $F_{1} x$ nach aufsteigenden Potenzen von $x$. Dividirt man dann $F x$ durch $F_{1} x$, so findet man

ebenso

$$
F x=\left(\alpha_{1}+\beta_{1} x\right) F_{1} x-x^{2} \stackrel{2}{F} x ;
$$

$$
F_{1} x=\left(\alpha_{2}+\beta_{2} x\right) \stackrel{2}{F} x-x^{2} \stackrel{3}{F} x
$$


und so weiter. Diese Reihe von Functionen, welche gleichmässig mit $\stackrel{m}{\boldsymbol{F}} x$ schliesst, hat ebenfalls die Eigenschaft, dass zwischen $a$ und $b$ so viel reelle Wurzeln der gabenen Gleichung liegen, als die Differenz der Anzahl der Zeichenwechsel in den zwei Reihen $\boldsymbol{F} a, F_{1} a, \stackrel{2}{F} a \ldots \stackrel{m}{\boldsymbol{F}} a$ und $\boldsymbol{F} b, F_{1} b, \stackrel{2}{F} b \ldots \stackrel{m}{\boldsymbol{F}} b$ beträgt.

Berücksichtigt man bloss die algebraischen Gleichungen, so möchte dieses zweite Verfahren schwerlich dem ersten vorzuziehen sein. Dagegen hat es den Vortheil, dass es leicht auf die Auflösung der transcendenten Gleichungen übertragen werden kann. Es bedarf hierzu nur einer kleinen Modification, ähnlich derjenigen, durch welche auch die Fourier'sche Methode auf transcendente Gleichungen anwendbar wird*). Man wird nämlich, wenn eine transcendente Gleichung aufgelöset werden soll, nicht mehr immer unmittelbar bestimmen können, wie viel reelle Wurzeln zwischen zwei beliebig gewählten Grenzen $a$ und $b$ liegen, sondern oft genöthigt sein, diesen $Z$ wischenraum erst

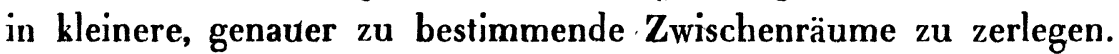

Es sei nämlich die transcendente Gleichung

$$
F x=a_{0}+a_{1} x+a_{2} x^{2}+\ldots=0
$$

gegeben, also $F_{1} x=a_{1}+2 a_{2} x+\ldots$

Dividirt man $F x$ durch $F_{1} x$, so ergiebt sich

$$
\boldsymbol{F} x=\left(\alpha_{1}+\beta_{1} x\right) F_{1} x-x^{2} \stackrel{2}{F} x .
$$

Eben so ergiebt sich aus der Division von $F_{1} x$ durch $F_{x}$ :

$$
\boldsymbol{F}_{1} x=\left(\alpha_{2}+\beta_{2} x\right) \stackrel{2}{\boldsymbol{F}} x-x^{2} \mathbf{F} x \text {. }
$$

Durch Fortsetzung dieses Verfahrens findet man also eine Functionenreihe $F_{x}, F_{1} x, \stackrel{2}{F} x, \stackrel{3}{F} x \ldots$, die jedoch nicht, wie bei den algebraischen Gleichungen, abbricht, sondern beliebig fortgesetzt werden kann. Man bleibe aber bei irgend einer abgeleiteten Function $\stackrel{r}{\boldsymbol{F}} \boldsymbol{x}$ stehen. Da diese Function, wie $F \boldsymbol{x}$, zwischen gewissen Grenzen continuirlich sein muss, so wird man immer zwei Zahlen, $m$ und $n>m$, finden können, so beschaffen, dass $\stackrel{r}{F} x$ zwischen den Grenzen $x=m$ und $x=n$ dasselbe Zeichen behält. Alsdann hat man folgenden Satz:

Die transcendente Gleichung hat eben so viele reelle Wurzeln, deren Werthe zwischen $m$ und $n$ liegen, als die Differenz der Anzahl der

*) Vergl. Bd. 22. Seite 14. dieses Journals. 
Zeichenwechsel in den zwei Reihen $F m, F_{1} m \ldots F^{r} m$ und $F_{n}$ $F_{1} n \ldots . \stackrel{r}{F} n$ beträgt.

Der Beweis ist eben so wie bei den algebraischen Gleichungen zu führen und bedarf keiner weitern Erläuterung.

Es sei z. B. die Gleichung

$$
F x=1-x+\frac{x^{2}}{(1.2)^{2}}-\frac{x^{3}}{(1.2 .3)^{2}}+\frac{x^{4}}{(1.2 .3 .4)^{2}}-\ldots=0
$$

gegeben. Hier ist

und daher

$$
F_{1} x=-1+\frac{x}{1.2}-\frac{x^{2}}{(1.2)^{2} .3}+\frac{x^{3}}{(1.2 .3)^{2} .4}-\ldots
$$

$$
\stackrel{2}{F} x=\frac{1}{2^{2} \cdot 3}-\frac{x}{2^{2} \cdot 3.4}+\frac{x^{2}}{2^{3} .3 .4 .5}-\frac{x^{2}}{2^{3} \cdot 3^{2} .4 .5 \cdot 6}+\ldots
$$

Das allgemeine Glied der letzteren Reihe ist

$$
\frac{ \pm \frac{1}{2} x^{m}}{2^{2} \cdot 3^{2} \ldots m^{2} \cdot m+1 \cdot m+2 \cdot m+3}
$$

und man findet aus diesem Gliede das folgende, indiem man es mit $\frac{x}{m+1 . m+4}$ multiplicirt. Sobald $x<4$ ist, wird mithin jedes positive Glied der Reihe grösser als das folgende negative sein, d. h. $\stackrel{2}{\boldsymbol{F}} x$ wird zwischen den Grenzen $x=0$ und $x=4$ immer positiv sein. Man setze daher $r=2$, so kann man nun bestimmen, wie viele reelle Wurzeln der Gleichung zwischen $x=0$ und $x=4$ liegen. Die Functionen $F x, F_{1} x, \mathbb{F}_{x}$ geben nämlich für $x=0$ die Zeichenreihe +-+ , für $x=4$ die Zeichenreihe -++ , also liegt eine reelle Wurzel zwischen 0 und 4. 